\title{
CLOSED FORM SOLUTION TO SOME MIXED BOUNDARY VALUE PROBLEMS FOR A CHARGED SPHERE
}

\author{
V. I. FABRIKANT ${ }^{1}$
}

(Received 15 May 1985; revised 24 March 1986)

\begin{abstract}
A new method is described which allows an exact solution in a closed form to the following non-axisymmetric mixed boundary-value problem for a charged sphere: arbitrary potential values are given at the surface of a spherical segment while an arbitrary charge distribution is prescribed on the rest of the sphere. The method is founded on a new integral representation of the kernel of the governing integral equation. Several examples are considered. All the results are expressed in elementary functions. Some further applications of the method are discussed. No similar result seems to have been published previously.
\end{abstract}

\section{Introduction}

The usual method of treatment of mixed boundary value problems in potential theory involves expansion of the solution in series of spherical harmonics. The convergence of such solutions depends heavily on the smoothness of the boundary conditions. In many practical applications, the boundary conditions are discontinuous, which renders some such solutions practically useless due to a very weak convergency close to the surface of the sphere and even divergency at its surface. The usual way out of this situation is to find some ingenious method to present the solution in the form of a sum of a closed form expression and a series expansion with a good convergence. All this makes it very important to find an exact closed-form solution to the mixed boundary value problem for a sphere.

Reviewing the literature, we could not find a general closed-form solution even for an axisymmetric mixed problem. An extensive list of references can be found in books $[4,5,6]$. Some mixed problems for a spherical segment are solved in [6]

\footnotetext{
${ }^{1}$ Department of Mechanical Engineering, Concordia University, Montreal, Quebec H3G 1M8, Canada. (C) Copyright Australian Mathematical Society 1987, Serial-fee code 0334-2700/87
} 
by means of the Mehler-Fok integral transform, but the method does not allow any mixed conditions at the spherical surface of the segment; only at the planar part of the segment the normal derivative can be specified.

An exact solution in closed form is obtained here to the following mixed problem for a sphere: an arbitrary potential value is given at a spherical segment, and an arbitrary charge density is prescribed on the rest of the sphere. Some preliminaries, helping to understand the method, are given in the next section, after which the general solution follows. Two examples are considered: i) a constant potential is prescribed at the surface of a spherical cap, and a uniform charge density on the rest; ii) the potential value is assumed to be proportional to the $z$-coordinate, and the charge density to the $x$-coordinate.

\section{Preliminaries}

Certain quantities are introduced in this section to simplify understanding of the new approach. The following two integrals will be used in this paper, namely

$$
\begin{aligned}
& \int \frac{\lambda\left(x^{2} / p q, \beta\right) d x}{\sqrt{p^{2}-x^{2}} \sqrt{q^{2}-x^{2}}}=-\left(1 / R_{p q}\right) \tan ^{-1}\left(y_{1}(x) / R_{p q}\right), \\
& \int \frac{\lambda\left(p q / x^{2}, \beta\right) d x}{\sqrt{x^{2}-p^{2}} \sqrt{x^{2}-q^{2}}}=\left(1 / R_{p q}\right) \tan ^{-1}\left(y_{2}(x) / R_{p q}\right) .
\end{aligned}
$$

Here

$$
\begin{gathered}
\lambda(k, \beta)=\frac{1-k^{2}}{1+k^{2}-2 k \cos \beta}=\sum_{n=-\infty}^{\infty} k^{|n|} e^{i n \beta}, \\
y_{1}(x)=\sqrt{p^{2}-x^{2}} \sqrt{q^{2}-x^{2}} / x, \\
y_{2}(x)=\sqrt{x^{2}-p^{2}} \sqrt{x^{2}-q^{2}} / x, \\
R_{p q}^{2}=p^{2}+q^{2}-2 p q \cos \beta .
\end{gathered}
$$

Correctness of the integrals ( 1 and 2 ) can be verified by direct substitution of ( 4 and 5 ) into (1 and 2 ) respectively.

The following integral $L$-operator will also be used

$$
\begin{aligned}
L(k) f(\phi) & =\frac{1}{2 \pi} \int_{0}^{2 \pi} \lambda(k, \phi-\psi) f(\psi) d \psi \\
& =\sum_{n=-\infty}^{\infty} k^{|n|} e^{i n \phi} \frac{1}{2 \pi} \int_{0}^{2 \pi} e^{-i n \psi} f(\psi) d \psi \\
& =\sum_{n=-\infty}^{\infty} k^{|n|} f_{n} e^{i n \phi},
\end{aligned}
$$


where $f_{n}$ is the Fourier coefficient of the function $f$. The $L$-operator has the following properties from (7)

$$
L(k) L\left(k_{1}\right)=L\left(k k_{1}\right), \quad L(1) f=f
$$

and as a consequence, the operator inverse to the $L$-operator, is

$$
L^{-1}(k)=L\left(k^{-1}\right) \text {. }
$$

\section{Formulation of the problem and its solution}

Introduce the set of spherical coordinates $(r, \theta, \phi)$. Consider the following mixed boundary-value problem: find the electrostatic field of a charged sphere of radius $a$ when the charge density $q$ is given at the surface of a spherical segment while an arbitrary potential value $v$ is prescribed at the rest of the sphere.

The electrostatic field potential of a charged sphere can be presented in the form

$$
\begin{aligned}
V(r, \theta, \phi)= & a^{2} \int_{0}^{2 \pi} d \phi_{0} \int_{0}^{\alpha} R^{-1} q\left(\theta_{0}, \phi_{0}\right) \sin \theta_{0} d \theta_{0} \\
& +a^{2} \int_{0}^{2 \pi} d \phi_{0} \int_{\alpha}^{\pi} R^{-1} q\left(\theta_{0}, \phi_{0}\right) \sin \theta_{0} d \theta_{0}
\end{aligned}
$$

where

$$
R^{2}=r^{2}+a^{2}-2 r a\left[\cos \theta \cos \theta_{0}+\sin \theta \sin \theta_{0} \cos \left(\phi-\phi_{0}\right)\right] .
$$

Introduce the notations

$$
\begin{aligned}
& \quad \eta=2 a \tan (\theta / 2), \quad \eta_{0}=2 a \tan \left(\theta_{0} / 2\right), \\
& l_{1,2}\left(r, a, \theta, \theta_{0}\right) \\
& =\frac{1}{2}\left[\sqrt{a^{2}+r^{2}-2 a r \cos \left(\theta+\theta_{0}\right)} \mp \sqrt{a^{2}+r^{2}-2 a r \cos \left(\theta-\theta_{0}\right)}\right] .
\end{aligned}
$$

The geometrical interpretation of these notations is obvious. The following properties of $l_{1}$ and $l_{2}$ should be noted:

$$
l_{1} l_{2}=r a \sin \theta \sin \theta_{0} ; \quad l_{1}^{2}+l_{2}^{2}=r^{2}+a^{2}-2 a r \cos \theta \cos \theta_{0}
$$

so that

$$
R^{2}=l_{1}^{2}+l_{2}^{2}-2 l_{1} l_{2} \cos \left(\phi-\phi_{0}\right)
$$

The last expression can be rewritten in terms of $\eta$ and $\eta_{0}$ in the following manner:

$$
R^{2}=\xi^{2}\left[\eta^{2}+\eta_{0}^{2}-2 \eta \eta_{0} \cos \left(\phi-\phi_{0}\right)+\zeta^{2}\right]
$$


where

$$
\xi^{2}=16 a^{3} r /\left[\left(4 a^{2}+\eta^{2}\right)\left(4 a^{2}+\eta_{0}^{2}\right)\right], \quad \zeta^{2}=(a-r)^{2} / \xi^{2} .
$$

Introducing the notations

$$
k_{1,2}\left(\eta_{0}\right)=\frac{1}{2}\left[\sqrt{\left(\eta+\eta_{0}\right)^{2}+\zeta^{2}} \mp \sqrt{\left(\eta-\eta_{0}\right)^{2}+\zeta^{2}}\right],
$$

expression (14) can be rewritten as

$$
R^{2}=\xi^{2}\left[k_{1}^{2}\left(\eta_{0}\right)+k_{2}^{2}\left(\eta_{0}\right)-2 k_{1}\left(\eta_{0}\right) k_{2}\left(\eta_{0}\right) \cos \left(\phi-\phi_{0}\right)\right] .
$$

Here the following easily verifiable properties were used:

$$
k_{1} k_{2}=\eta \eta_{0}, \quad k_{1}^{2}+k_{2}^{2}=\eta^{2}+\eta_{0}^{2}+\zeta^{2}, \quad l_{1,2}=\xi k_{1,2}
$$

Now formulae (1-6) allow us to construct the following integral representations:

$$
\begin{aligned}
& \frac{1}{R}=\frac{2}{\pi \xi} \int_{0}^{k_{1}\left(\eta_{0}\right)} \frac{\lambda\left(x^{2} /\left(\eta \eta_{0}\right), \phi-\phi_{0}\right) d x}{\sqrt{k_{1}^{2}\left(\eta_{0}\right)-x^{2}} \sqrt{k_{2}^{2}\left(\eta_{0}\right)-x^{2}}} ; \\
& \frac{1}{R}=\frac{2}{\pi \xi} \int_{k_{2}\left(\eta_{0}\right)}^{\infty} \frac{\lambda\left(\eta \eta_{0} / x^{2}, \phi-\phi_{0}\right) d x}{\sqrt{x^{2}-k_{1}^{2}\left(\eta_{0}\right)} \sqrt{x^{2}-k_{2}^{2}\left(\eta_{0}\right)}} .
\end{aligned}
$$

Using the properties (18), expressions (19) and (20) can be rewritten in the following form:

$$
\begin{aligned}
& \frac{1}{R}=\frac{2}{\pi \xi} \int_{0}^{k_{1}\left(\eta_{0}\right)} \frac{\lambda\left(x^{2} /\left(\eta \eta_{0}\right), \phi-\phi_{0}\right) d x}{\sqrt{\eta^{2}-m^{2} x^{2}} \sqrt{\eta_{0}^{2}-g^{2}(x)}} \\
& \frac{1}{R}=\frac{2}{\pi \xi} \int_{k_{2}\left(\eta_{0}\right)}^{\infty} \frac{\lambda\left(\eta \eta_{0} / x^{2}, \phi-\phi_{0}\right) d x}{\sqrt{m^{2} x^{2}-\eta^{2}} \sqrt{g^{2}(x)-\eta_{0}^{2}}}
\end{aligned}
$$

where

$$
\begin{gathered}
m^{2}=1+\beta^{2} / 4 a^{2}, \quad \beta^{2}=(a-r)^{2}\left(4 a^{2}+\eta^{2}\right) / 4 a r, \\
g^{2}(x)=x^{2}\left|\frac{\eta^{2}+\beta^{2}-x^{2}}{\eta^{2}-m^{2} x^{2}}\right| .
\end{gathered}
$$

One can notice that the function $g$ is inverse to $k_{1}$ for $0 \leqslant x<\eta / m$, and is inverse to $k_{2}$ for $x^{2} \geqslant \eta^{2}+\beta^{2}$.

Substitution of (21) and (22) into the first and the second terms of (10) respectively yields, after the change of the order of integration:

$$
\begin{aligned}
W(r, \eta, \phi)= & 4 \int_{0}^{k_{1}(b)} \frac{d x}{\sqrt{\eta^{2}-m^{2} x^{2}}} \int_{g(x)}^{b} \frac{\eta_{0} d \eta_{0}}{\sqrt{\eta_{0}^{2}-g^{2}(x)}} L\left(\frac{x^{2}}{\eta \eta_{0}}\right) \sigma\left(\eta_{0}, \phi\right) \\
& +4 \int_{k_{2}(b)}^{\infty} \frac{d x}{\sqrt{m^{2} x^{2}-\eta^{2}}} \int_{b}^{g(x)} \frac{\eta_{0} d \eta_{0}}{\sqrt{g^{2}(x)-\eta_{0}^{2}}} L\left(\frac{\eta \eta_{0}}{x^{2}}\right) \sigma\left(\eta_{0}, \phi\right) .
\end{aligned}
$$


Here

$$
\begin{gathered}
b=2 a \tan (\alpha / 2), \quad \sigma\left(\eta_{0}, \phi_{0}\right)=8 a^{3} q\left(2 \tan ^{-1}\left(\eta_{0} / 2 a\right), \phi_{0}\right)\left(4 a^{2}+\eta_{0}^{2}\right)^{-3 / 2} \\
W(r, \eta, \phi)=2 \sqrt{a r} V\left(r, 2 \tan ^{-1}(\eta / 2 a), \phi\right) / \sqrt{4 a^{2}+\eta^{2}}
\end{gathered}
$$

The quanities $\sigma$ and $W$ were introduced in such a way that in the limiting case $a \rightarrow \infty$ they turn into the charge density and the potential as the sphere turns into a half-space. We recall that the $L$-operator in (25) is understood according to its definition (7).

It is appropriate now to split our problem into two: i) to find the electrostatic field potential of a charged sphere when the zero potential is prescribed at the surface of a spherical segment, and an arbitrary change density is given at the rest of the sphere; ii) to find the electrostatic field potential of a charged sphere when an arbitrary potential value is prescribed at the surface of a spherical segment, and there is zero charge density elsewhere. Hereafter these problems are treated separately.

Problem 1:

Consider the potential problem of a charged sphere subject to the following boundary conditions at $r=a$ :

$$
\begin{gathered}
V(a, \theta, \phi)=0 \text { for } 0 \leqslant \phi<2 \pi, 0 \leqslant \theta<\alpha, \\
q=q(\theta, \phi) \text { for } 0 \leqslant \phi<2 \pi, \alpha<\theta \leqslant \pi .
\end{gathered}
$$

Substitution of the boundary conditions (28) in (25) leads to the following integral equation

$$
\begin{aligned}
0= & \int_{0}^{\eta} \frac{d x}{\sqrt{\eta^{2}-x^{2}}} \int_{x}^{b} \frac{\eta_{0} d \eta_{0}}{\sqrt{\eta_{0}^{2}-x^{2}}} L\left(\frac{x^{2}}{\eta \eta_{0}}\right) \sigma\left(\eta_{0}, \phi\right) \\
& +\int_{b}^{\infty} \frac{d x}{\sqrt{x^{2}-\eta^{2}}} \int_{b}^{x} \frac{\eta_{0} d \eta_{0}}{\sqrt{x^{2}-\eta_{0}^{2}}} L\left(\frac{\eta \eta_{0}}{x^{2}}\right) \sigma\left(\eta_{0}, \phi\right) .
\end{aligned}
$$

It is important to notice that $\sigma$ in the first term of (29) is yet unknown while the value of $\sigma$ in the second term of (29) is known from (28) and (26). It is then necessary to express one through the other. Using the integral representation (21) instead of (22), expressions (29) can be rewritten as follows:

$$
\begin{aligned}
& \int_{0}^{\eta} \frac{d x}{\sqrt{\eta^{2}-x^{2}}} \int_{x}^{b} \frac{\eta_{0} d \eta_{0}}{\sqrt{\eta_{0}^{2}-x^{2}}} L\left(\frac{x^{2}}{\eta \eta_{0}}\right) \sigma\left(\eta_{0}, \phi\right) \\
& =-\int_{0}^{\eta} \frac{d x}{\sqrt{\eta^{2}-x^{2}}} \int_{b}^{\infty} \frac{\eta_{0} d \eta_{0}}{\sqrt{\eta_{0}^{2}-x^{2}}} L\left(\frac{x^{2}}{\eta \eta_{0}}\right) \sigma\left(\eta_{0}, \phi\right),
\end{aligned}
$$


with an immediate result

$$
\int_{x}^{b} \frac{\eta_{0} d \eta_{0}}{\sqrt{\eta_{0}^{2}-x^{2}}} L\left(\frac{x}{\eta_{0}}\right) \sigma\left(\eta_{0}, \phi\right)=-\int_{b}^{\infty} \frac{\eta_{0} d \eta_{0}}{\sqrt{\eta_{0}^{2}-x^{2}}} L\left(\frac{x}{\eta_{0}}\right) \sigma\left(\eta_{0}, \phi\right) .
$$

Application of the operator

$$
L(\eta) \frac{d}{d \eta} \int_{\eta}^{b} \frac{x d x}{\sqrt{x^{2}-\eta^{2}}} L\left(\frac{1}{x}\right)
$$

to both sides of (30) results in

$$
\sigma(\eta, \phi)=-\frac{2}{\pi} \frac{1}{\sqrt{b^{2}-\eta^{2}}} \int_{b}^{\infty} \frac{\sqrt{\eta_{0}^{2}-b^{2}}}{\eta_{0}^{2}-\eta^{2}} L\left(\frac{\eta}{\eta_{0}}\right) \sigma\left(\eta_{0}, \phi\right) \eta_{0} d \eta_{0}
$$

Returning to the spherical coordinates and the original notations, one gets

$$
\begin{aligned}
q(\theta, \phi)= & -\frac{1}{2 \pi^{2}} \frac{1}{\sqrt{\cos \theta-\cos \alpha}} \\
& \times \int_{0}^{2 \pi} \int_{\alpha}^{\pi} \frac{\sqrt{\cos \alpha-\cos \theta_{0}}}{1-\cos \theta \cos \theta_{0}-\sin \theta \sin \theta_{0} \cos \left(\phi-\phi_{0}\right)},
\end{aligned}
$$

which corresponds to the source function discovered in geometrical form by Lord Kelvin who used his method of images. In the case of axial symmetry, expression (32) simplifies, after integration with respect to $\phi_{0}$, to

$$
q(\theta)=-\frac{1}{\pi} \frac{1}{\sqrt{\cos \theta-\cos \alpha}} \int_{\alpha}^{\pi} \frac{\sqrt{\cos \alpha-\cos \theta_{0}} q\left(\theta_{0}\right) \sin \theta_{0} d \theta_{0}}{\cos \theta-\cos \theta_{0}} .
$$

Now the charge density is defined all over the sphere, and the electrostatic field potential can be expressed directly through $q$ by substitution of (31) in the first term of (25). Changing the order of integration and the integration with respect to $\eta_{0}$ gives

$$
\begin{aligned}
W(r, \eta, \phi)= & -4 \int_{0}^{k_{1}(b)} \frac{d x}{\sqrt{\eta^{2}-m^{2} x^{2}}} \int_{b}^{\infty} \frac{y d y}{\sqrt{y^{2}-g^{2}(x)}} L\left(\frac{x^{2}}{\eta y}\right) \sigma(y, \phi) \\
& +4 \int_{k_{2}(b)}^{\infty} \frac{d x}{\sqrt{m^{2} x^{2}-\eta^{2}}} \int_{b}^{g(x)} \frac{\eta_{0} d \eta_{0}}{\sqrt{g^{2}(x)-\eta_{0}^{2}}} L\left(\frac{\eta \eta_{0}}{x^{2}}\right) \sigma\left(\eta_{0}, \phi\right) .
\end{aligned}
$$

The second term in (34) can be presented, using (21), as

$$
4 \int_{b}^{\infty} \eta_{0} d \eta_{0}\left\{\int_{0}^{k_{1}\left(\eta_{0}\right)} \frac{d x}{\sqrt{\eta^{2}-m^{2} x^{2}} \sqrt{\eta_{0}^{2}-g^{2}(x)}} L\left(\frac{x^{2}}{\eta \eta_{0}}\right)\right\} \sigma\left(\eta_{0}, \phi\right)
$$


Now one can change the order of integration in the following manner:

$$
\int_{b}^{\infty} d \eta_{0} \int_{0}^{k_{1}\left(\eta_{0}\right)} d x=\int_{0}^{k_{1}(b)} d x \int_{b}^{\infty} d \eta_{0}+\int_{k_{1}(b)}^{k_{1}(\infty)} d x \int_{g(x)}^{\infty} d \eta_{0}
$$

and this allows one to rewrite (35) as

$$
\begin{aligned}
& 4 \int_{0}^{k_{1}(b)} \frac{d x}{\sqrt{\eta^{2}-m^{2} x^{2}}} \int_{b}^{\infty} \frac{\eta_{0} d \eta_{0}}{\sqrt{\eta_{0}^{2}-g^{2}(x)}} L\left(\frac{x^{2}}{\eta \eta_{0}}\right) \sigma\left(\eta_{0}, \phi\right) \\
& \quad+4 \int_{k_{1}(b)}^{k_{1}(\infty)} \frac{d x}{\sqrt{\eta^{2}-m^{2} x^{2}}} \int_{g(x)}^{\infty} \frac{\eta_{0} d \eta_{0}}{\sqrt{\eta_{0}^{2}-g^{2}(x)}} L\left(\frac{x^{2}}{\eta \eta_{0}}\right) \sigma\left(\eta_{0}, \phi\right) .
\end{aligned}
$$

Substitution of (36) in (34) yields, considering that $k_{1}(\infty)=\eta / m$,

$$
W(r, \eta, \phi)=4 \int_{k_{1}(b)}^{\eta / m} \frac{d x}{\sqrt{\eta^{2}-m^{2} x^{2}}} \int_{g(x)}^{\infty} \frac{\eta_{0} d \eta_{0}}{\sqrt{\eta_{0}^{2}-g^{2}(x)}} L\left(\frac{x^{2}}{\eta \eta_{0}}\right) \sigma\left(\eta_{0}, \phi\right) .
$$

A change of the order of integration in (37) and the integration with respect to $x$ results in

$$
W(r, \eta, \phi)=\frac{2}{\pi} \int_{0}^{2 \pi} d \phi_{0} \int_{b}^{\infty} \frac{1}{R_{0}} \tan ^{-1}\left(\frac{\kappa}{R_{0}}\right) \sigma\left(\eta_{0}, \phi_{0}\right) \eta_{0} d \eta_{0}
$$

where

$$
R_{0}^{2}=\eta^{2}+\eta_{0}^{2}-2 \eta \eta_{0} \cos \left(\phi-\phi_{0}\right)+\zeta^{2},
$$

and the value of $\kappa$ can be defined in several ways, namely

$$
\begin{aligned}
\kappa & =\sqrt{k_{1}^{2}\left(\eta_{0}\right)-k_{1}^{2}(b)} \sqrt{k_{2}^{2}\left(\eta_{0}\right)-k_{1}^{2}(b)} / k_{1}(b) \\
& =\sqrt{\eta_{0}^{2}-b^{2}} \sqrt{k_{2}^{2}(b)-b^{2} m^{2}} / b \\
& =\sqrt{\eta_{0}^{2}-b^{2}} \sqrt{\eta^{2}-k_{1}^{2}(b) m^{2}} / k_{1}(b) .
\end{aligned}
$$

Returning to spherical coordinates, one gets

$$
V(r, \theta, \phi)=\frac{2}{\pi} \int_{0}^{2 \pi} d \phi_{0} \int_{\alpha}^{\pi} \frac{q\left(\theta_{0}, \phi_{0}\right)}{R} \tan ^{-1} \frac{\chi}{R} a^{2} \sin \theta_{0} d \theta_{0},
$$

where

$$
R^{2}=a^{2}+r^{2}-2 a r\left[\cos \theta \cos \theta_{0}+\sin \theta \sin \theta_{0} \cos \left(\phi-\phi_{0}\right)\right]
$$

and

$$
\chi=\xi_{\kappa}=\frac{\sqrt{2} \sqrt{\cos \alpha-\cos \theta_{0}} \sqrt{l_{2}^{2}(\alpha)-\sin ^{2} \frac{\alpha}{2} l_{2}^{2}(\pi)}}{\sin \alpha} .
$$


Here $l_{1,2}(x)$ stands as an abbreviation for $l_{1,2}(r, a, \theta, x)$, as defined by (12). The function

$$
\frac{2}{\pi} \frac{1}{R} \tan ^{-1} \frac{\chi}{R}
$$

can be interpreted as a source function. Formulae (37) and (41) give two equivalent expressions for the electrostatic field potential, the first one being more convenient for the exact evaluation of the integrals, while the second has definite advantages for numerical integration.

Problem 2:

Consider a sphere with the following boundary conditions at its surface $r=a$ :

$$
\begin{aligned}
V(a, \theta, \phi) & =v(\theta, \phi) \quad \text { for } 0 \leqslant \phi<2 \pi, 0 \leqslant \theta<\alpha, \\
q(\theta, \phi) & =0 \text { for } 0 \leqslant \phi<2 \pi, \alpha<\theta \leqslant \pi .
\end{aligned}
$$

Substitution of the boundary conditions (44) in (25) leads to the following integral equation:

$$
w(\eta, \phi)=4 \int_{0}^{\eta} \frac{d x}{\sqrt{\eta^{2}-x^{2}}} \int_{x}^{b} \frac{\eta_{0} d \eta_{0}}{\sqrt{\eta_{0}^{2}-x^{2}}} L\left(\frac{x^{2}}{\eta \eta_{0}}\right) \sigma\left(\eta_{0}, \phi\right),
$$

where $w$ is related to $v$ according to (27). An equation similar to (45) was treated in [2]. Here, a different type of solution is suggested. Application of the operator

$$
L\left(\frac{1}{t}\right) \frac{d}{d t} \int_{0}^{t} \frac{\eta d \eta}{\sqrt{t^{2}-\eta^{2}}} L(\eta)
$$

to both sides of (45) gives

$$
2 \pi \int_{t}^{b} \frac{\eta_{0} d \eta_{0}}{\sqrt{\eta_{0}^{2}-t^{2}}} L\left(\frac{t}{\eta_{0}}\right) \sigma\left(\eta_{0}, \phi\right)=L\left(\frac{1}{t}\right) \frac{d}{d t} \int_{0}^{t} \frac{\eta d \eta}{\sqrt{t^{2}-\eta^{2}}} L(\eta) w(\eta, \phi) .
$$

The second operator to apply is

$$
\frac{L(y)}{y} \frac{d}{d y} \int_{y}^{b} \frac{t d t}{\sqrt{t^{2}-y^{2}}} L\left(\frac{1}{t}\right)
$$

The result is

$$
\sigma(y, \phi)=-\frac{1}{\pi^{2} y} L(y) \frac{d}{d y} \int_{y}^{b} \frac{t d t}{\sqrt{t^{2}-y^{2}}} L\left(\frac{1}{t^{2}}\right) \frac{d}{d t} \int_{0}^{t} \frac{\eta d \eta}{\sqrt{t^{2}-\eta^{2}}} L(\eta) w(\eta, \phi) .
$$

Expression (46) can be presented in another form, using the rules of differentiation under the integral sign and the properties of the $L$-operator (8):

$$
\sigma(y, \phi)=\frac{1}{\pi^{2}}\left[\frac{\Phi(b, y, \phi)}{\sqrt{b^{2}-y^{2}}}-\int_{y}^{b} \frac{d t}{\sqrt{t^{2}-y^{2}}} \frac{\partial}{\partial t} \Phi(t, y, \phi)\right],
$$


where

$$
\Phi(t, y, \phi)=\frac{1}{t} \int_{0}^{t} \frac{\eta d \eta}{\sqrt{t^{2}-\eta^{2}}} \frac{\partial}{\partial \eta}\left[\eta L\left(\frac{\eta y}{t^{2}}\right) w(\eta, \phi)\right] .
$$

Integration by parts allows to establish the following identity:

$$
\frac{\partial}{\partial t} \Phi(t, y, \phi)=\int_{0}^{t} \frac{\eta d \eta}{\sqrt{t^{2}-\eta^{2}}} L\left(\frac{\eta y}{t^{2}}\right) \Delta w(\eta, \phi) .
$$

Here $\Delta$ is the two-dimensional Laplace operator in the polar coordinates. Now the solution (47) can be rewritten as follows:

$$
\sigma(y, \phi)=\frac{1}{\pi^{2}}\left[\frac{\Phi(b, y, \phi)}{\sqrt{b^{2}-y^{2}}}-\int_{y}^{b} \frac{d t}{\sqrt{t^{2}-y^{2}}} \int_{0}^{t} \frac{\eta d \eta}{\sqrt{t^{2}-\eta^{2}}} L\left(\frac{\eta y}{t^{2}}\right) \Delta w(\eta, \phi)\right] .
$$

A further simplification is possible by changing of the order of integration in (50) and integration with respect to $t$. The result is

$$
\begin{aligned}
& \sigma(y, \phi)=\frac{1}{\pi^{2}}\left[\frac{\Phi(b, y, \phi)}{\sqrt{b^{2}-y^{2}}}-\frac{1}{2 \pi} \int_{0}^{2 \pi} \int_{0}^{b} \frac{\Delta w\left(\eta, \phi_{0}\right) \eta d \eta d \phi_{0}}{\sqrt{y^{2}+\eta^{2}-2 \eta y \cos \left(\phi-\phi_{0}\right)}}\right. \\
& \left.\times \tan ^{-1} \frac{\sqrt{b^{2}-\eta^{2}} \sqrt{b^{2}-y^{2}}}{b \sqrt{y^{2}+\eta^{2}-2 \eta y \cos \left(\phi-\phi_{0}\right)}}\right] .
\end{aligned}
$$

The expression for the charge density (51) consists of two distinct terms: the first one is singular at the edge $(y \rightarrow b$ ) while the second tends to zero as $y \rightarrow b$.

Now it is possible to express the electrostatic field potential directly through the given value of $v(\theta, \phi)$. The substitution of (46) in (25) gives, after simplification,

$$
\begin{aligned}
W(r, \eta, \phi)= & \frac{2}{\pi} \int_{0}^{k_{1}(b)} \frac{d x}{\sqrt{\eta^{2}-m^{2} x^{2}}} L\left(\frac{x^{2}}{\eta g^{2}(x)}\right) \\
& \times \frac{\partial}{\partial g(x)} \int_{0}^{g(x)} \frac{\eta_{0} d \eta_{0}}{\sqrt{g^{2}(x)-\eta_{0}^{2}}} L\left(\eta_{0}\right) w\left(\eta_{0}, \phi\right) .
\end{aligned}
$$

Here the following property of the Abel-type operators was used:

$$
\int_{g}^{a} \frac{d r}{\sqrt{r^{2}-g^{2}}} \frac{d}{d r} \int_{r}^{a} \frac{t f(t) d t}{\sqrt{t^{2}-r^{2}}}=-\frac{\pi}{2} f(g)
$$


Introduction of the new variable $u=g(x), x=k_{1}(u)$ allows to rewrite (52) as $W(r, \eta, \phi)=\frac{2}{\pi} \int_{0}^{b} \frac{d k_{1}(u)}{\sqrt{\eta^{2}-m^{2} k_{1}^{2}(u)}} L\left(\frac{\eta}{k_{2}^{2}(u)}\right) \frac{\partial}{\partial u} \int_{0}^{u} \frac{\eta_{0} d \eta_{0}}{\sqrt{u^{2}-\eta_{0}^{2}}} L\left(\eta_{0}\right) w\left(\eta_{0}, \phi\right)$.

A change of the order of integration in (54) gives

$$
\begin{aligned}
W(r, \eta, \phi)= & \frac{2}{\pi} \int_{0}^{b} d \eta_{0} L\left(\eta_{0}\right) w\left(\eta_{0}, \phi\right) \frac{d}{d \eta_{0}} \\
& \times \int_{\eta_{0}}^{b} \frac{u d k_{1}(u)}{\sqrt{\eta^{2}-m^{2} k_{2}^{2}(u)} \sqrt{u^{2}-\eta_{0}^{2}}} L\left(\frac{\eta}{k_{2}^{2}(u)}\right) .
\end{aligned}
$$

The $u$-integration in (55) can be performed to give, after returning to the spherical coordinates:

$$
V(r, \theta, \phi)=\frac{a\left|r^{2}-a^{2}\right|}{2 \pi^{2}} \int_{0}^{2 \pi} d \phi_{0} \int_{0}^{\alpha}\left(\frac{R}{\chi_{1}}+\tan ^{-1} \frac{\chi_{1}}{R}\right) \frac{v\left(\theta_{0}, \phi_{0}\right)}{R^{3}} \sin \theta_{0} d \theta_{0},
$$

where $R$ is defined by (42), and

$$
\chi_{1}=\frac{\sqrt{2} \sqrt{\cos \theta_{0}-\cos \alpha} \sqrt{l_{2}^{2}(\alpha)-\cos ^{2} \frac{\alpha}{2} l_{2}^{2}(0)}}{\sin \alpha} .
$$

One can notice that in the case $\alpha \rightarrow \pi$ formula (56) transforms into the well-known Poisson's solution to the Dirichlet problem for a sphere. In the limiting case of $r \rightarrow a$, formula (56) gives the potential value at the rest of the sphere through its value prescribed at the segment $\theta \leqslant \alpha$ :

$$
\begin{aligned}
& V(a, \theta, \phi)=\frac{\sqrt{\cos \alpha-\cos \theta}}{2 \pi^{2}} \\
& \quad \times \int_{0}^{2 \pi} d \phi_{0} \int_{0}^{\alpha} \frac{v\left(\theta_{0}, \phi_{0}\right) \sin \theta_{0} d \theta_{0}}{\sqrt{\cos \theta_{0}-\cos \alpha}\left[1-\cos \theta \cos \theta_{0}-\sin \theta \sin \theta_{0} \cos \left(\phi-\phi_{0}\right)\right]} .
\end{aligned}
$$

A further simplification becomes possible in the case of axial symmetry, namely

$$
V(a, \theta)=\frac{\sqrt{\cos \alpha-\cos \theta}}{\pi} \int_{0}^{2 \pi} \int_{0}^{\alpha} \frac{v\left(\theta_{0}\right) \sin \theta_{0} d \theta_{0}}{\sqrt{\cos \theta_{0}-\cos \alpha}\left(\cos \theta_{0}-\cos \theta\right)} .
$$

Notice certain similarity between formulae (32-33) and (58-59). Expressions (54) and (56) give two equivalent forms of solution to the problem, the first formula being more convenient for exact evaluation of the integrals. 


\section{Illustrative examples}

Example 1: Consider a charged sphere with the following conditions at $r=a$, $0 \leqslant \phi \leqslant \pi$ :

$$
\begin{aligned}
& V(a, \theta, \phi)=v_{0}=\text { const. for } 0 \leqslant \theta<\alpha \text {; } \\
& q(\theta, \phi)=q_{0}=\text { const. for } \alpha<\theta \leqslant \pi \text {. }
\end{aligned}
$$

The charge density at the surface of the spherical cap $0 \leqslant \theta<\alpha$ is given by superposition of the results of evaluation of (33) and (46), and is

$$
\begin{aligned}
q(\theta)= & \frac{v_{0}}{2 \pi^{2} a}\left[\frac{\sqrt{1+\cos \alpha}}{\sqrt{\cos \theta-\cos \alpha}}+\tan ^{-1} \sqrt{\frac{\cos \theta-\cos \alpha}{1+\cos \alpha}}\right] \\
& -\frac{2}{\pi} q_{0}\left[\frac{\sqrt{1+\cos \alpha}}{\sqrt{\cos \theta-\cos \alpha}}-\tan ^{-1} \sqrt{\frac{1+\cos \alpha}{\cos \theta-\cos \alpha}}\right] .
\end{aligned}
$$

One can notice that for $q_{0}=v_{0} / 4 \pi a, q(\theta)=q_{0}$ with an obvious interpretation.

The potential distribution can be obtained from (37) and (54). The result of integration is

$$
\begin{aligned}
V(r, \theta)= & \frac{2}{\pi} v_{0}\left[\frac{a+r}{2 r} \sin ^{-1} A_{1}-\frac{|a-r|}{2 r} \sin ^{-1} A_{2}\right] \\
& +4 a q_{0}\left[\frac{a+r}{2 r} \cos ^{-1} A_{1}-\frac{|a-r|}{2 r} \cos ^{-1} A_{2}\right],
\end{aligned}
$$

where

$$
\begin{aligned}
& A_{1}=(a+r) \sin (\alpha / 2) / \sqrt{l_{2}^{2}(\alpha)+4 a r \sin ^{2}(\alpha / 2) \sin ^{2}(\theta / 2)} \\
& A_{2}=|a-r| \sin (\alpha / 2) / \sqrt{l_{2}^{2}(\alpha)-4 a r \sin ^{2}(\alpha / 2) \cos ^{2}(\theta / 2)}
\end{aligned}
$$

Again, in the case of $q_{0}=v_{0} / 4 \pi a$, one gets the potential distribution of a uniformly charged sphere

$$
V(r)=\frac{v_{0}}{2 r}[a+r-|a-r|] .
$$

Example 2: Consider a non-axisymmetric problem when a potential value, proportional to the $z$-coordinate, is given at a spherical segment, and the charge density, proportional to the $x$-coordinate, is prescribed on the rest of the sphere. The boundary conditions are formulated as follows:

$$
\begin{aligned}
V(a, \theta, \phi) & =v_{0} \cos \theta, \quad 0 \leqslant \theta<\alpha, \\
q(\theta, \phi) & =q_{0} \sin \theta \cos \phi, \quad \alpha<\theta \leqslant \pi .
\end{aligned}
$$


Formulae (37) and (54) give, after integration,

$$
\begin{gathered}
V(r, \theta, \phi)=\frac{2 v_{0}}{\pi r}\left\{\left[\frac{r^{3}+a^{3}}{2 a r} \sin ^{-1} A_{1}-\frac{\left|r^{3}-a^{3}\right|}{2 a r} \sin ^{-1} A_{2}\right] \cos \theta\right. \\
\left.+\frac{\sqrt{l_{2}^{2}(\alpha)-\sin ^{2} \frac{\alpha}{2} l_{2}^{2}(\pi)}}{\sin (\alpha / 2)}\left[A_{1}^{2} \sin ^{2} \frac{\theta}{2}-A_{2}^{2} \cos ^{2} \frac{\theta}{2}\right]\right\} \\
+\frac{4 q_{0} a}{3 r} \sin \theta \cos \phi\left\{\left[\frac{r^{3}+a^{3}}{2 a r} \cos ^{-1} A_{1}-\frac{\left|r^{3}-a^{3}\right|}{2 a r} \cos ^{-1} A_{2}\right]\right. \\
\left.+\frac{\sqrt{l_{2}^{2}(\alpha)-\sin ^{2} \frac{\alpha}{2} l_{2}^{2}(\pi)}}{\sin (\alpha / 2)}\left[A_{1}^{2}+A_{2}^{2}\right]\right\},
\end{gathered}
$$

where $A_{1}$ and $A_{2}$ are defined by (63). Certain simplification occurs at the surface of the sphere where the potential value is $v_{0} \cos \theta$ for $0 \leqslant \theta<\alpha$, and for $\alpha<\theta \leqslant \pi$

$$
\begin{aligned}
V(a, \theta, \phi)= & \frac{2}{\pi} v_{0}\left[\cos \theta \sin ^{-1} t_{1}+\sqrt{1-\cos \alpha} \sqrt{\cos \alpha-\cos \theta}\right] \\
& +\frac{8}{3} a q_{0} \sin \theta \cos \phi\left[\cos ^{-1} t_{1}+t_{1} \sqrt{1-t_{1}^{2}}\right],
\end{aligned}
$$

where $t_{1}=\sin (\alpha / 2) / \sin (\theta / 2)$. The charge density at $0 \leqslant \theta<\alpha$ can be obtained from (33) and (46), and is

$$
\begin{aligned}
q(\theta, \phi)= & \frac{v_{0}}{2 \pi^{2} a}\left[\frac{\sqrt{2} \cos \frac{3}{2} \alpha}{\sqrt{\cos \theta-\cos \alpha}}+3 \sqrt{2} \cos \frac{\alpha}{2} \sqrt{\cos \theta-\cos \alpha}\right. \\
& \left.+3 \cos \theta \tan ^{-1} \frac{\cos \theta-\cos \alpha}{1+\cos \alpha}\right] \\
& -\frac{2}{\pi} q_{0} \sin \theta \cos \phi\left[\frac{1-t_{2}^{2} / 3}{\sqrt{t_{2}^{2}-1}}-\tan ^{-1} \frac{1}{\sqrt{t_{2}^{2}-1}}\right],
\end{aligned}
$$

where $t_{2}=\cos (\theta / 2) / \cos (\alpha / 2)$. The total charge $Q$ can be evaluated as

$$
Q=\left(v_{0} a / \pi\right) \sin \alpha(1+\cos \alpha),
$$

and consequently the potential value at the centre $r=0$ is

$$
V(0)=\left(v_{0} / \pi\right) \sin \alpha(1+\cos \alpha) \text {. }
$$




\section{Discussion}

It is of interest to compare the results of this paper with those available in the literature. Expression (56) can be considered as a generalization for a spherical cap of the well known Poisson's formula for a sphere. One can indicate an alternative method of derivation of (56), by differentiation of the Green's function for a spherical cap obtained by Hobson [3]. All the other major results of this paper seem to be quite new.

The practical use of expressions of the type (41) and (56) is very limited, except for direct numerical integration, due to the complexity of the Green's functions under the integral sign. On the other hand, expressions (37) and (54) are equivalent to (41) and (56), and are very convenient for practical evaluation of the integrals involved, as it is shown by the examples considered in previous section.

The problem of a spherical cap at a constant potential was considered by several authors. The list of references and the solution can be found in [1], and is, in our notation,

$$
V(r, \theta)=\left(v_{0} / \pi r\right)\left[r \gamma+a \gamma^{\prime}\right]
$$

where

$$
\frac{\sin \gamma}{a}=\frac{\sin \gamma^{\prime}}{r}=\frac{\sin \alpha}{l_{2}(\alpha)}
$$

The angles $\gamma$ and $\gamma^{\prime}$ are discontinuous at the surface of the cap, and may assume the values between 0 and $\pi / 2$ or between $\pi / 2$ and $\pi$ depending where the point $(r, \theta)$ is, which constitutes the main difficulty in the practical use of $(68)$. One can show that the first term in (62) is equivalent to (68), and is free of the above mentioned inconveniences, since the inverse trigonometric functions are understood everywhere as their principal values.

The solution to the problem of spherical cap, held at the potential $v=v_{0} \cos \theta$, can be rewritten from [1] as follows

$$
\begin{aligned}
V(r, \theta)= & \left(v_{0} / \pi a\right)\left[\left(\gamma+a^{3} \gamma^{\prime} / r^{3}\right) r \cos \theta+(a \cos \alpha-r \cos \theta) \tan \gamma\right. \\
& \left.+r^{-2} a^{2}(r \cos \alpha-a \cos \theta) \tan \gamma^{\prime}\right],
\end{aligned}
$$

where $\gamma$ and $\gamma^{\prime}$ are defined by (69), which indicates the same difficulties in the practical use of (70). Again, one can show that (70) is equivalent to the term in the first two lines of (65).

Notice that certain integral characteristics can be found without completely solving the problem. For example, if one needs the value of the total charge $Q$ in the Problem 1, there is no need to compute (32). Direct integration under the 
integral sign gives

$$
Q=\frac{2}{\pi} \int_{0}^{2 \pi} d \phi \int_{\alpha}^{\pi} q(\theta, \phi) \cos ^{-1} \frac{\sin (\alpha / 2)}{\sin (\theta / 2)} \sin \theta d \theta
$$

The method presented can be successfully used to solve some mixed problems for several spheres or spherical caps, and can be generalised for the case of more than three dimensions. Notice also that for the case $a \rightarrow \infty$ all the results of this paper can be interpreted as the exact solution to the mixed boundary value problem for a half-space.

\section{Acknowledgements}

This investigation was supported by research grants from the Natural Sciences and Engineering Research Council of Canada.

\section{References}

[1] W. D. Collins, "On the solution of some axisymmetric boundary value problems by means of integral equations", Quart. J. Mech. Appl. Math. 12 pt. 2, (1959), 232-241.

[2] V. I. Fabrikant and T. S. Sankar, "On contact problems in inhomogeneous half-space", Internat. J. Soltds and Structures 20 (1984), 159-166.

[3] E. W. Hobson, "On Green's function for a circular disc, with application to electrostatic problems", Trans. Cambridge Phil. Soc. 18 (1900), 277-291.

[4] A. I. Lur'e, Three-dimensional problems of the theory of elasticity (Interscience Publishers, New York, 1964).

[5] I. N. Sneddon, Mixed boundary value problems in potential theory (North-Holland Publishing Company, Amsterdam, 1966).

[6] Ya. S. Uflyand, Method of dual equations in mathematical physics (Nauka, Leningrad, 1977) (in Russian). 